\title{
Ribosome Inactivation
}

National Cancer Institute

\section{Source}

National Cancer Institute. Ribosome Inactivation. NCI Thesaurus. Code C41602.

Processes that involve interference with, or restraint of, the activity of ribosomes. 